\title{
SOME EXPLICITLY INVERTIBLE TOEPLITZ OPERATORS ON THE QUARTER-PLANE
}

\author{
JAMES RADLOW
}

\begin{abstract}
A 2-variable Toeplitz operator $T$ is invertible only under special conditions on its symbol $t(z) \equiv t\left(z_{1}, z_{2}\right)$. Here we make three additions to the known list of such special conditions and we construct explicit inverses in each of the three cases. The results are obtained by the use of a 2-variable Wiener-Hopf factorization of the symbol.
\end{abstract}

Let $T$ denote a Toeplitz operator on the quarter-plane. Let its symbol have the Laurent expansion

$$
t(z)=\sum t_{j} z^{j}
$$

where $j=\left(j_{1}, j_{2}\right)$ and $z=\left(z_{1}, z_{2}\right)$ is on the torus $T^{2}$, the Cartesian product $\left|z_{1}\right|=1 \times\left|z_{2}\right|=1$. Let $\left\{t_{j}\right\} \in l^{1}$. Then the Laurent expansion converges absolutely. The symbol $t(z)$ therefore belongs to $W^{2}$, the ring of absolutely convergent Fourier series on $T^{2}$. The $W^{2}$ norm (sum of the moduli of the Fourier coefficients) equals the $l^{1}$ norm.

Let $P_{q}(q=1,2,3,4)$ denote projection of $W^{2}$ into $W_{q}^{2}$, the subring of quarter-range Fourier series with coefficients indexed by lattice points $j=$ $\left(j_{1}, j_{2}\right)$ in the $q$ th quadrant. The quadrant is closed for $q=1$ and open for $q=2,3,4$. This gives $P_{1}+P_{2}+P_{3}+P_{4}=I$.

Just as the coefficients of an element of $W^{2}$ define a vector in $l^{1}$, so a series in $W_{q}^{2}$ corresponds to an element in the vector subspace $l_{q}^{1}$. If $f, g$ are vectors in $l_{1}^{1}$, we denote by $f(z), g(z)$ the corresponding Fourier series in $W_{1}^{2}$. We can represent the action of the Toeplitz operator $T$ on the quarter-plane by

$$
(T f)(z)=P_{1}(t(z) f(z))=g(z) .
$$

Since $P_{1}=I-\left(P_{2}+P_{3}+P_{4}\right)$, we can rewrite (1) as

$$
t(z) f(z)=g(z)+\sum_{j=2}^{4} h_{j}(z)
$$

where $h_{j}(z)=P_{j}(t f)$ for $j=2,3,4$. Equations (1), (2) hold on $T^{2}$.

A Toeplitz operator $T$ with zero-dimensional kernel and cokernel has an inverse. The invertibility problem for $T$ is equivalent to the function-

Received by the editors December 3, 1976.

AMS (MOS) subject classifications (1970). Primary 47B35, 45E10; Secondary 32A10, 42A92, $42 A 96$.

Key words and phrases. Toeplitz operator, symbol, two-variable Wiener-Hopf factorization, Fredholm operator. 
theoretical problem of solving (2) for the unknown function $f(z)$. On the circle such an equation as (2) is solved (Krĕin [3, §13]) by a Wiener-Hopf factorization of $t(z)$. The analogous two-variable factorization for symbols $t(z)$ which are nonzero on the torus and homotopic to a constant has the form

$$
t(z)=\prod_{q=1}^{4} t_{q}(z)
$$

where $t_{q}(z) \in W_{q}^{2}$. Malyšev [4] and Strang [8] remark that the factorization (3) does not appear to yield a solution of (2) on the torus, even when the inverse exists. It is known that a Toeplitz operator on the quarter-plane is Fredholm of index zero if and only if its symbol is nonzero on the torus and homotopic to a constant. (The proof was given by Simonenko [7] and independently by Douglas-Howe [2] for opertors on $l_{1}^{2}$, and by Strang [8] for operators on $l_{1}^{p}, p \geqslant 1$.) But Fredholmness is not invertibility. Further conditions on $t(z)$ are needed for invertibility: see for example Malyšev [4], [5], Osher [6], Douglas and Howe [2], and Douglas [1].

Here we consider symbols $t(z)$ possessing a factorization (3) and satisfying one of three special conditions. In each of the cases we construct an explicit inverse $T^{-1}$ by making use of (3). Before stating the result we note that the factorization/Fredholmness conditions $\left(t(z) \in W^{2}, t(z) \neq 0\right.$ on $T^{2}, t(z)$ homotopic to a constant) are both necessary and sufficient for the existence of (3). Under these conditions each factor $t_{q}(z)$ is holomorphic and nonzero in a domain $U_{q}^{2}$. We have: $U_{1}^{2}\left(\left|z_{1}\right|<1,\left|z_{2}\right|<1\right) ; U_{2}^{2}\left(\left|z_{1}\right|>1,\left|z_{2}\right|<1\right)$; $U_{3}^{2}\left(\left|z_{1}\right|>1,\left|z_{2}\right|>1\right)$; and $U_{4}^{2}\left(\left|z_{1}\right|<1,\left|z_{2}\right|>1\right)$.

THEOREM. Let the factorization (3) hold, and let $t_{q}(z)=1$ for: (a) $q=2$; (b) $q=4$; or (c) $q=2$ and $q=4$. Then the Toeplitz operator $T$ is invertible. The inverse operator $T^{-1}$ such that $\left(T^{-1} g\right)(z)=f(z)$, where $g(z)$ and $f(z)$ are series in $W_{1}^{2}$ related by (1), is

$$
\begin{aligned}
& T^{-1}=t_{1}^{-1} P_{1}\left[t_{4}^{-1}\left(P_{1}\right) t_{3}^{-1}\right], \\
& T^{-1}=t_{1}^{-1} P_{1}\left[t_{2}^{-1}\left(P_{1}\right) t_{3}^{-1}\right], \\
& T^{-1}=t_{1}^{-1}\left(P_{1}\right) t_{3}^{-1}
\end{aligned}
$$

in the respective cases (a), (b), (c).

Proof. Consider equation (2). By hypothesis: $t(z) \in W^{2}, f(z)$ and $g(z) \in$ $W_{1}^{2}$, and $h_{j}(z) \in W_{j}^{2}$ for $j=2,3,4$. Equation (2) holds on $T^{2}$.

In case (a), $t_{2}(z)=1$. Since $t_{3}(z) \neq 0$ in $U_{3}^{2}$, we can multiply (2) by $t_{3}^{-1}$. We then project the result onto $W_{1}^{2} \cup W_{4}^{2}$ by means of $P_{R}=P_{1}+P_{4}$. Since $P_{R}\left[t_{3}^{-1}\left(h_{2}+h_{3}\right)\right]=0$, we have:

$$
t_{1} t_{4} f=P_{R}\left(t_{3}^{-1} g\right)+P_{R}\left(t_{3}^{-1} h_{4}\right)
$$

Next multiply (5) by $t_{4}^{-1}$ and project the result onto $W_{1}^{2}$ by means of $P_{1}$. Since $P_{1}\left[t_{4}^{-1} P_{4}\left(t_{3}^{-1} g\right)\right]=0$ and $P_{1}\left(t_{3}^{-1} h_{4}\right)=0$, we then have 


$$
t_{1} f=P_{1}\left[t_{4}^{-1} P_{1}\left(t_{3}^{-1} g\right)\right] .
$$

If we multiply (6) by $t_{1}^{-1}$, we arrive at the (clearly unique) result

$$
f(z)=t_{1}^{-1}(z) P_{1}\left[t_{4}^{-1}(z) P_{1}\left(t_{3}^{-1}(z) g(z)\right)\right] \text {. }
$$

We have shown that if $T^{-1}$ exists then $T f=g$ is solved by (7). To complete the proof in case (a), we must show that: (i) for any vector $g$ in $l_{1}^{1}$, (7) defines a series $f(z)$ in $W_{1}^{2}$; (ii) the vector $f$ in $l_{1}^{1}$ formed from the coefficients of that series $f(z)$ is a solution of the Toeplitz operator equation $T f=g$ in $l_{1}^{1}$.

We omit the (easy) proof of (i). To prove assertion (ii), we reverse the steps which led to (7). By so doing we find that (7) is equivalent to

$$
t f=g+t_{3}\left(R_{2}+R_{3}+R_{4}\right)
$$

where $R_{j}(z)$ is an unknown element of $W_{j}^{2}$. Then we project both sides of (8) onto $W_{1}^{2}$ by means of $P_{1}$. Since $P_{1}\left(t_{3} R_{j}\right)=0$ for $j=2,3,4$, this gives

$$
P_{1}(t(z) f(z))=g(z) \text {. }
$$

To show that (9) is equivalent to (8), we form the Cauchy product of the series $t(z)$ and $f(z)$, and then apply $P_{1}$ to the result. This completes the proof in case (a). The proof for case (b) follows by symmetry. Case (c) is a specialization of either (a) or (b).

The results are easily extended to Toeplitz operators on $l_{1}^{p}, p \geqslant 1$.

\section{REFERENCES}

1. R. G. Douglas, On the invertibility of a class of Toeplitz operators on the quarter-plane, Indiana Univ. Math. J. 21 (1972), 1031-1035.

2. R. G. Douglas and R. Howe, On the $C^{*}$-algebra of Toeplitz operators on the quarter-plane, Trans. Amer. Math. Soc. 158 (1971), 203-217.

3. M. G. Krein, Integral equations on a half-line with kernel depending upon the difference of the arguments, Uspehi Mat. Nauk 13 (1958), no. 5 (83), 3-120; English transl., Amer. Math. Soc. Transl. (2) 22 (1962), 163-288. MR 21 \# 1507.

4. V. A. Malyšev, On the solution of discrete Wiener-Hopf equations in a quarter-plane, Dokl. Akad. Nauk SSSR 187 (1969), 1243-1246 = Soviet Math. Dokl. 10 (1969), 1032-1036.

5. Wiener-Hopf equations in a quadrant, discrete groups, and automorphic functions, Mat. Sb. 84 (1971), 499-525.

6. S. J. Osher, On certain Toeplitz operators in two variables, Pacific J. Math. 34 (1970), 123-129.

7. I. B. Simonenko, Multidimensional discrete convolution operators, Math. Issled. (Kishinev) 3 (1968), 108-122.

8. Gilbert Strang, Toeplitz operators in a quarter-plane, Bull. Amer. Math. Soc. 76 (1970), 1303-1307.

Department of Mathematics, University of New Hampshire, Durham, New Hampshire 03824 